\title{
Near-surface viscosity effects on capillary rise of water in nanotubes
}

\author{
Truong Quoc Vo, ${ }^{1}$ Murat Barisik, ${ }^{2, *}$ and BoHung Kim ${ }^{1, \dagger}$ \\ ${ }^{1}$ School of Mechanical Engineering, University of Ulsan, Daehak-ro 93, Namgu, Ulsan 680-749, South Korea \\ ${ }^{2}$ Department of Mechanical Engineering, Izmir Institute of Technology, Urla, Izmir 35430, Turkey \\ (Received 26 January 2015; revised manuscript received 26 May 2015; published 11 November 2015)
}

\begin{abstract}
In this paper, we present an approach for predicting nanoscale capillary imbibitions using the Lucas-Washburn (LW) theory. Molecular dynamics (MD) simulations were employed to investigate the effects of surface forces on the viscosity of liquid water. This provides an update to the modified LW equation that considered only a nanoscale slip length. An initial water nanodroplet study was performed to properly elucidate the wetting behavior of copper and gold surfaces. Intermolecular interaction strengths between water and corresponding solid surfaces were determined by matching the contact angle values obtained by experimental measurements. The migration of liquid water into copper and gold capillaries was measured by MD simulations and was found to differ from the modified LW equation. We found that the liquid layering in the vicinity of the solid surface induces a higher density and viscosity, leading to a slower MD uptake of fluid into the capillaries than was theoretically predicted. The near-surface viscosity for the nanoscale-confined water was defined and calculated for the thin film of water that was sheared between the two solid surfaces, as the ratio of water shear stress to the applied shear rate. Considering the effects of both the interface viscosity and slip length of the fluid, we successfully predicted the MD-measured fluid rise in the nanotubes.
\end{abstract}

DOI: 10.1103/PhysRevE.92.053009

PACS number(s): 47.55.nb, 68.03.Kn, 47.35.Pq, 66.20.-d

\section{INTRODUCTION}

The capillary action and nanowetting of solid surfaces in porous media or narrow cylindrical capillaries are observed in many micro- and nanofluidic systems from numerous industrial and medical processes; such as enhanced oil recovery and nanodrug delivery [1-3]. Capillary imbibitions result in the motion of the meniscus, which is formed between the liquid and its vapor, and is attached to the wall by a contact line. This fluid motion is driven by a pressure drop that occurs across the liquid-vapor interface. Regardless of the well-studied capillary behavior on both macro- and microscales, the rise of fluid in nanometer confinements having at least one dimension on the order of $100 \mathrm{~nm}$ or smaller [4] has yet to be fully characterized. Nanoscale capillary imbibitions are indispensable, making the understanding and control of their rapid evolution one of the most critical tasks in the field of material science.

In the 1910s, Lucas and Washburn effectively expressed macroscopic-scale fluid penetration in a straight-line capillary $(H)$ as a function of the square root of time $(t)$. This is called the Lucas-Washburn (LW) equation [5,6]:

$$
H=\sqrt{\frac{\gamma_{\mathrm{LV}} R \cos \theta}{2 \eta}} \sqrt{t},
$$

where $R$ is the pore radius, $\gamma_{\mathrm{LV}}$ is the liquid-vapor surface tension, $\eta$ is the liquid viscosity, and $\theta$ is the contact angle (CA) between the liquid meniscus and the capillary wall. The classical hypothesis of the LW equation (1) was derived from the Stokes equation of momentum, based on Poiseuille flow with no-slip boundary assumptions, in which the capillary force is balanced by the viscous drag force. However, when the system size decreases from macrolevels

\footnotetext{
*Corresponding author: muratbarisik@iyte.edu.tr

†bohungk@ulsan.ac.kr
}

to microns, submicrons, or nanometers, the fluid dynamics significantly differs from that of macroscales making first the no-slip boundary condition and subsequently all continuum descriptions invalid [7].

Studies of nanoscale capillaries have recently shown a notable deviation from the LW predictions, observed as an enhanced uptake of fluid into capillaries compared to molecular dynamics (MD) results [8-13]. Researchers have concluded that this difference is due to the no-slip boundary condition assumed in the LW equation. Depending on the wettability of the solid surface, the slip lengths reaching the range of 0-20 $\mathrm{nm}$ are comparable with the characteristic dimensions of such small confinements [7]. In 2008, Schebarchov and Hendy [14] modified the LW equation (1) in order to explicitly consider a Navier slip length $(b)$ for small scales as follows:

$$
H=\sqrt{\frac{\gamma_{\mathrm{LV}}(R+4 b) \cos \theta}{2 \eta}} \sqrt{t} .
$$

However, consideration of the boundary slip does not entirely explain nanoscale capillary behaviors. The continuum assumption fails in nanoscale systems since the molecular motion of discrete molecules is anisotropically constrained by the wall-fluid and fluid-fluid interactions. The surface effects dominate the nanoscale conduits as the force penetration distance induced by the intermolecular force fields of the wall molecules becomes comparable to the confinement dimensions. Thus, the molecular structures of the fluid and solid wall, as well as their interactions at atomistic length scales, play key roles in studying nanoscale fluid dynamics [15-23].

The layering of fluid molecules near solid surfaces is a well-known phenomenon. This dynamic structuring occurs as the thin liquid film flows in a series of distinct molecular layers. The structured liquid layers are not prominent in most macroscopic problems. However, they are important in multiscale methods or nanoscale fluid flow problems, in which 
the continuum descriptions completely break down, and the dynamics depends on the variations on the interface or on the flow boundary layer [24,25]. Furthermore, these solidlike liquid layers influence fluid properties, resulting in a different shear rate or viscosity at the wall-fluid boundary, as well as in the channel center [4,18-20]. However, these nanoscale effects on capillary action of liquid water apparently have not been characterized to date.

Viscosity typically resists the flow of fluids, and therefore it is expected to affect the capillary imbibitions of nanoconfined fluids strongly. The relationship between the bulk viscosity and the slip length was previously introduced as $b=\eta / k$, where friction $k$ is an interfacial property [26]. However, recent experimental studies have revealed the significant influence of an apparent viscosity on capillary filling speed $[4,27]$. For example, the disagreement between the theoretical calculations and measurements was found to result from a nearsurface viscosity that was inducing higher apparent viscosity values than the known bulk viscosity values. Additionally, Tas et al. [4] suggested two origins for this high apparent viscosity: (1) the electroviscous effect due to the electroosmotic counterflow induced by a streaming potential [28]; and (2) an increase in polar water molecules near the solid walls [29].

Given these challenges, there is a need to investigate the limitations of the continuum hypothesis for nanoscale fluid flow, especially the effects of near-surface viscosity. In such cases, a better estimation of capillary action at the nanoscale can be performed by the proper consideration of molecular behaviors at the solid-liquid boundary. Therefore, our objective was to provide an approach based on Eq. (2) by considering the effects of both Navier slip length and interface viscosity to effectively predict the fluid rise in cylindrical capillaries comprised of copper and gold materials.

This paper is organized as follows: (1) in Sec. II, we summarize the details of the MD simulations of liquid water in a nanopore, a water droplet on a solid substrate, liquid water in a periodic cubic box, and a liquid water film sheared between two solid walls. (2) In Sec. III, the calculation details of the LW equation (2), contact angle, liquid-vapor surface tension, bulk viscosity, and Navier slip length are described. Specifically, the MD capillary imbibitions are shown with a comparison with the predictions of Eq. (2). In addition, the possible reasons for the slower migration of fluid into the nanoscale capillaries are discussed. Furthermore, the influences of interface viscosity and our proposed approach for improved capillary imbibition prediction are presented. (3) Finally, our findings and conclusions are reported.

\section{MOLECULAR SIMULATION DETAILS}

\section{A. Water model}

Water models can be classified by the number of points used to define the water structure, and whether the structure is treated as rigid. For simplicity and computational efficiency, we chose the simple point charge-extend (SPC-E) water model for this study. The SHAKE algorithm was applied to constrain the $\mathrm{O}-\mathrm{H}$ bond length of $0.1 \mathrm{~nm}$ and the $\mathrm{H}-\mathrm{O}-\mathrm{H}$ angle of $109.47^{\circ}$, thereby making the model rigid [30]. The
TABLE I. Details of the molecular parameters utilized in this study.

\begin{tabular}{lclcc}
\hline \hline Interaction & $q(\mathrm{e})$ & $\sigma(\mathrm{nm})$ & $\varepsilon(\mathrm{eV})$ & $\varepsilon_{\mathrm{r}}$ \\
\hline $\mathrm{H}-\mathrm{H}$ & 0.4238 & 0 & 0 & \\
$\mathrm{O}-\mathrm{O}$ & -0.8476 & 0.3166 & 0.006739 & \\
$\mathrm{Cu}-\mathrm{O}$ & & 0.2751 & $\varepsilon_{\mathrm{r}} \varepsilon_{\mathrm{O}-\mathrm{O}}$ & $1.1-3.2$ \\
$\mathrm{Au}-\mathrm{O}$ & & 0.36 & $\varepsilon_{\mathrm{r}} \varepsilon_{\mathrm{O}-\mathrm{O}}$ & $1.1-3.2$ \\
\hline \hline
\end{tabular}

model can be described as effective rigid pair potentials composed of Lennard-Jones (L-J) and Coulombic terms [31]. The water molecular parameters are provided in Table I. The partial charges $q_{\mathrm{H}}=0.4238 e$ and $q_{\mathrm{O}}=-0.8476 e$ were assigned to hydrogen and oxygen atoms, respectively. The electrostatic interaction was modeled by using Coulomb's law. Therefore, the particle-particle, particle-mesh (PPPM) method was utilized to minimize the errors in long-range terms [32]. The truncated (12-6) Lennard-Jones (LJ) potential is given as

$$
\begin{aligned}
V_{\text {truncated }}= & 4 \varepsilon\left\{\left[\left(\frac{\sigma}{r_{i j}}\right)^{12}-\left(\frac{\sigma}{r_{i j}}\right)^{6}\right]\right. \\
& \left.-\left[\left(\frac{\sigma}{r_{c}}\right)^{12}-\left(\frac{\sigma}{r_{c}}\right)^{6}\right]\right\},
\end{aligned}
$$

where $\varepsilon$ is the depth of the potential well, $r_{i j}$ is the intermolecular distance, $\sigma$ is the finite molecular distance at which the interatomic potential is zero, and $r_{c}$ is the cutoff distance. In this study, a cutoff distance of $1.0 \mathrm{~nm}$ was applied.

\section{B. Water flow into the capillary}

Figure 1(a) describes the simulation domain of fluid migration into a cylindrical nanotube. We utilized a capillary length of $L=10 \mathrm{~nm}$ and a radius of $R=1 \mathrm{~nm}$ for all simulations. A total of 13050 liquid water molecules were initially kept in a rectangular $9 \times 9 \mathrm{~nm}^{2}$ reservoir to maintain an equilibrium state with its vapor by utilizing an $N V T$ ensemble. Various solids (i.e., copper and gold) were used to model the capillary rise of liquid water into the nanopores. Periodic boundary conditions were applied in the $X$ and $Y$ directions, perpendicular to the pore axis. In addition, both ends of the capillary simulation box were fixed by two artificial solid layers, which were modeled utilizing $\mathrm{Cu}$ or $\mathrm{Au}$, depending on the particular case. The atoms in these two layers were secured to their original locations to maintain a fixed volume system. Specifically, the top end of the cylindrical pore was enclosed with a hypothetical impenetrable wall, to prevent water molecules escaping from the pore. In contrast, another solid layer was positioned at the bottom end of the simulation box, $10 \mathrm{~nm}$ from the solid layer, parallel to the $X Y$ plane at the capillary entrance. This was to ensure that there were no effects of this layer on the liquid film or the capillary imbibitions. As a result, there exists a free liquid surface in the reservoir due to the limited number of water molecules.

Additionally, the (12-6) LJ potential was utilized to model the interactions between the wall and the oxygen atoms of the liquid water. The solid-liquid intermolecular diameter $\sigma_{\mathrm{W}-\mathrm{F}}$ can be calculated by the Lorentz-Berthelot (LB) mixing rules [32] given as $\sigma_{\mathrm{W}-\mathrm{F}}=\left(\sigma_{\mathrm{W}-\mathrm{W}}+\sigma_{\mathrm{F}-\mathrm{F}}\right) / 2$, where $\sigma_{\mathrm{W}-\mathrm{W}}$ and $\sigma_{\mathrm{F}-\mathrm{F}}$ are 


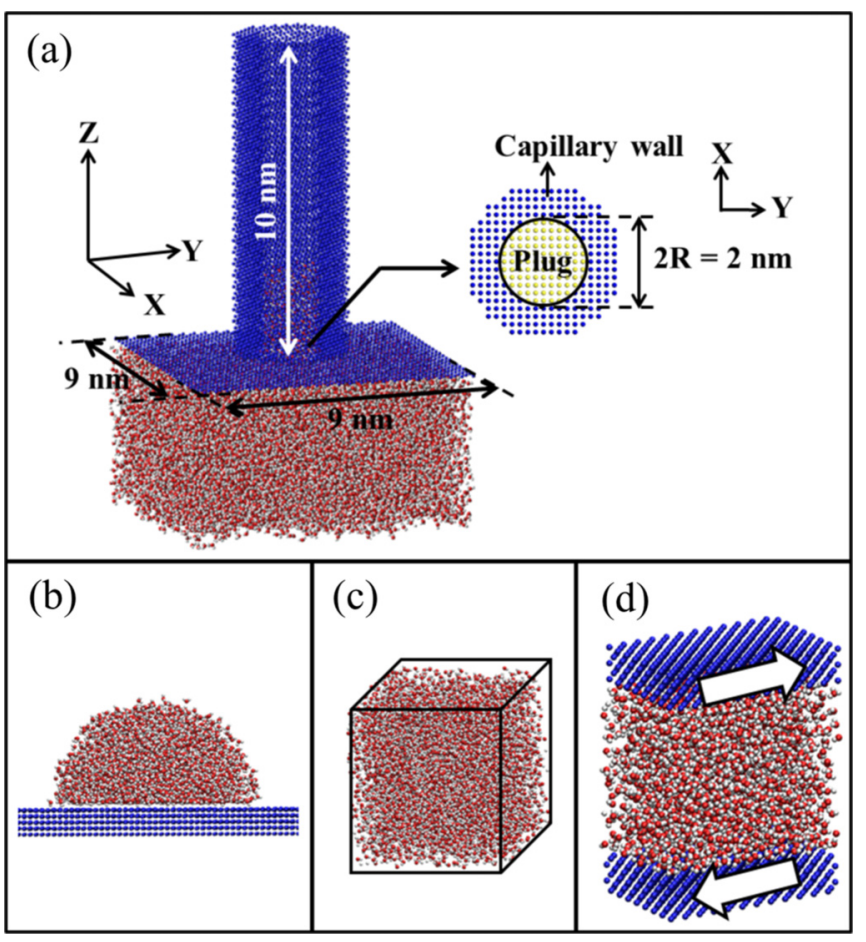

FIG. 1. (Color online) (a) Schematic simulation domains of liquid water uptake into a nanoscale capillary tube. (b) Water droplet on a solid substrate. (c) Liquid water molecules in a periodic cubic box. (d) Thin film of liquid water sheared between two solid walls.

the wall-wall and fluid-fluid molecular lengths, respectively. Moreover, we varied the wall-fluid interaction strength $\varepsilon_{\mathrm{W}-\mathrm{F}}$ as a function of the fluid-fluid interaction $\varepsilon_{\mathrm{F}-\mathrm{F}}$ as $\varepsilon_{\mathrm{W}-\mathrm{F}}=\varepsilon_{\mathrm{r}} \times \varepsilon_{\mathrm{F}-\mathrm{F}}$, where $\varepsilon_{\mathrm{r}}$ is the modulated coefficient or interaction strength ratio ranging from 1.1 to 3.2. All molecular parameters used in this study are presented in Table I. The embedded atom method was applied to model the interatomic forces between the solids. This is because it accurately describes the total energy of a metal by considering the embedding energy as a function of atomic electron density [33].

To ensure that the water molecules maintained a specific temperature while entering the tube, we used the dissipative particle dynamics (DPD) thermostat [34]. The dynamics of DPD particles is governed by Newton's second law $F=m \ddot{r}$, where $m$ is the mass of the DPD particle, and $r$ is the particle position. The DPD thermostat denotes the pairwise interactions between the particles via three terms given as $F=F^{\mathrm{C}}+F^{\mathrm{D}}+F^{\mathrm{R}}$. The conservation force in standard DPD reads $F^{\mathrm{C}}=A \omega(r)$, where $A$ is the repulsive force, and the weight function $\omega(r)=\left(1-r / r_{\mathrm{c}}\right)$ varies between 0 and 1 . The dissipative term is calculated as $F^{\mathrm{D}}=-\gamma \omega^{2}(r)\left(\hat{r}_{i j} \cdot \hat{v}_{i j}\right)$, where $v_{i j}$ is the relative velocity normal to the connecting line between particles. The random force reads $F^{\mathrm{R}}=$ $p \omega(r) g \tau^{-1 / 2}$, where $g$ is a Gaussian random number with a zero mean and unit variance, and $\tau$ is the size of the time step. The random and dissipative forces act as a thermostat if the amplitudes $p$ and the dissipative coefficient $\gamma$ satisfy the fluctuation-dissipation theorem $p=\sqrt{2 k_{\mathrm{B}} T \gamma}$, where $k_{\mathrm{B}}$ is the Boltzmann constant, and $T$ is the applied temperature. The DPD thermostat adequately preserves the momentum and hydrodynamics. Additionally, it satisfies the fluctuationdissipation theorem by rescaling particle velocities using pairwise interactions with neighboring particles [35]. These advantages of the DPD thermostat cannot be found when applying either a local thermostat (e.g., Langevin) or a global thermostat (e.g., Nosé-Hoover) [34,36], which result in a slower imbibition of liquid molecules [8].

Newton's equations of motion were integrated using the VERLET algorithm with a simulation time step $(\tau)$ of 1.0 fs. LAMMPS [37] was used for all MD simulations in the present study. Simulations were initiated from the MaxwellBoltzmann velocity distribution for all molecules at $300 \mathrm{~K}$ for 100 ps. The NVT ensemble and Nosé-Hoover thermostat were applied to maintain the system at a temperature of $300 \mathrm{~K}$. During this period, we utilized a plug at the capillary entrance to prevent water molecules from entering the pore. Therefore, the system reached a state of equilibrium. From this point, the DPD thermostat was employed to maintain the system at a constant temperature of $T=300 \mathrm{~K}$ with the $N V E$ ensemble for a period of $0.7 \mathrm{~ns}$. The pairwise interactions between the plug atoms and water molecules were simultaneously turned off at the beginning of the $0.7 \mathrm{~ns}$ time period. Thus, the uptake of water molecules into the capillary was taken into account.

\section{Water droplets on metallic substrates, water in a periodic cubic box, and thin film liquid water sheared between solid walls}

Water droplets on metallic substrates were modeled to investigate the surface wetting behaviors and to compute the liquid-vapor surface tension. These $C A$ values were considered to be the wetting angles between the capillary walls and the fluid menisci. Figure 1(b) describes the simulation domain of a nanoscale water droplet on a solid substrate with a thickness of two unit cells, in which the lattice constants of copper and gold are $3.61 \AA$ and $4.08 \AA$, respectively. A water droplet with a density of approximately $1.0 \mathrm{~g} / \mathrm{cm}^{3}$ was formed by randomly distributing 2197 water molecules throughout the simulation cell. We ensured that the simulation domain size was large enough to prevent any effect on the droplet. The liquid-vapor surface tension was calculated using the formula [38]

$$
\gamma_{\mathrm{LV}}=\frac{L_{Z}}{2}\left[\left\langle S_{Z Z}\right\rangle-\frac{1}{2}\left(\left\langle S_{X X}\right\rangle+\left\langle S_{Y Y}\right\rangle\right)\right],
$$

where $L_{Z}$ is the droplet height; and $S_{X X}, S_{Y Y}$, and $S_{Z Z}$ are the normal stress tensors. Details of the calculations for stress tensor components are provided in the further discussion.

In contrast, 4197 water molecules in a periodic cubic box of $5 \times 5 \times 5 \mathrm{~nm}^{3}$ dimensions, as seen in Fig. 1(c), corresponded to a density of $0.9984 \mathrm{~g} / \mathrm{cm}^{3}$. These molecules were modeled to study the bulk liquid water viscosity based on the GreenKubo formula. This formula relates the ensemble average of the auto-correlation of the stress or pressure tensor to the viscosity [39].

To calculate the Navier slip length and the local viscosity, we conducted MD simulations of shear-driven flow. Liquid water molecules were simulated in a three-dimensional channel with a height of $4 \mathrm{~nm}$, a width of $2.89 \mathrm{~nm}$, and a length of $4.82 \mathrm{~nm}$, as shown in Fig. 1(d). The wall velocity was set to $50 \mathrm{~nm} / \mathrm{ns}$. There is a common method for measuring viscosity that utilizes the stress tensor, including in molecular-level 
descriptions [40]. Recently, using this method, Kim et al. [18] and Vo et al. [19] computed the fluid viscosity, which showed excellent consistency with both other MD simulations and experiments [41]. The local dynamic viscosity of the liquid in the molecular system is defined as the ratio of the shear component of the stress tensor $\left(S_{X Z}\right)$ and the applied shear rate $(\dot{\gamma})$ in fluid mechanics as

$$
\eta=-\frac{\left\langle S_{X Z}\right\rangle}{\dot{\gamma}},
$$

where the stress tensor is calculated in the water domain.

Computations of the atomistic stress tensor included the two additive components of (1) kinetic and (2) virial terms $[42,43]$. For instance, the components of the stress tensor for atom $i$ are given by the following formula [44]:

$$
\begin{aligned}
S_{\alpha \beta}= & -\left(m v_{\alpha} v_{\beta}+\frac{1}{2} \sum_{n=1}^{N_{p}}\left(r_{1 \alpha} F_{1 \beta}+r_{2 \alpha} F_{2 \beta}\right)\right. \\
& +\frac{1}{2} \sum_{n=1}^{N_{b}}\left(r_{1 \alpha} F_{1 \beta}+r_{2 \alpha} F_{2 \beta}\right) \\
& +\frac{1}{3} \sum_{n=1}^{N_{a}}\left(r_{1 \alpha} F_{1 \beta}+r_{2 \alpha} F_{2 \beta}+r_{3 \alpha} F_{3 \beta}\right) \\
& \left.+K_{\text {space }}\left(r_{i \alpha}, F_{i \beta}\right)+\sum_{n=1}^{N_{f}} r_{i \alpha} F_{i \beta}\right),
\end{aligned}
$$

where the first term on the right-hand side is the kinetic component, $m$ is the atomic mass of particle $I$, and $v_{\alpha}$ and $v_{\beta}$ are the velocity components of particle $i$ in the $\alpha$ and $\beta$ directions, respectively. The second to fifth terms are virial components consisting of the following: (1) the second term is a pairwise energy contribution where $n$ loops over the $N_{p}$ neighbors of atom $i, r_{1}$ and $r_{2}$ are the positions, and $F_{1}$ and $F_{2}$ are the forces of the two atoms in the pairwise interaction; (2) the third and the fourth terms are the bond and angle contributions for the $N_{b}$ bonds, and the $N_{a}$ angle of which atom $i$ is part, respectively; (3) the $K_{\text {space }}$ term is the contribution from the long-range Coulombic interactions for the PPPM solver; and (4) the fifth term is the SHAKE internal constraint force applied to particle $i$ via the $N_{f}$ fixes. The per atom array values listed above are the products of the stress and volume units. Therefore, the local stress in each slab bin $\left(S_{\alpha \beta}^{\text {bin }}\right)$ positioned parallel to the walls is achieved by dividing the average of the total per atom stress tensor by the particular volume of the slab bin $\left(V_{\text {bin }}\right)$ as

$$
S_{\alpha \beta}^{\mathrm{bin}}=\left(S_{\alpha \beta} N_{\text {bin }}\right) / V_{\text {bin }},
$$

where $N_{\text {bin }}$ is the number of atoms in each slab bin.

It is important to note that the desired data were averaged after $1 \mathrm{~ns}$, when the systems reached an isothermal steady state using a 1 fs time step. The $N V T$ ensemble was applied via the Nosé-Hoover thermostat to maintain the systems at $300 \mathrm{~K}$. Moreover, the calculated slip length and viscosity from the shear-driven flow simulation could be utilized for general flow problems because the applied shear rate was in the linear regime [45].

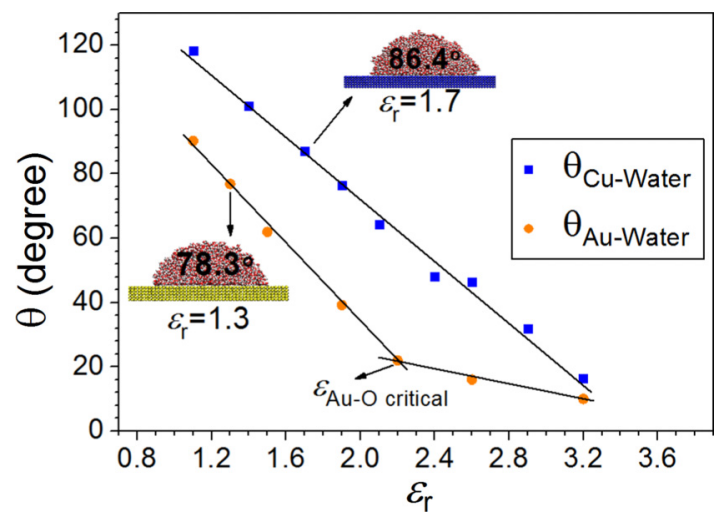

FIG. 2. (Color online) Contact angle variation as a function of wall-fluid interaction strength.

\section{RESULTS AND DISCUSSION}

\section{A. Surface wetting behaviors, surface tension, bulk viscosity, and slip length}

To study the wettability of solid surfaces, we measured the CA between the water droplets and solid substrates. The variations of water droplet CA with $\varepsilon_{\mathrm{r}}^{\mathrm{Cu}-\mathrm{O}}$ and $\varepsilon_{\mathrm{r}}^{\mathrm{Au}-\mathrm{O}}$ are plotted in Fig. 2. It was found that the CA linearly decreased with the strengthened $\varepsilon_{\mathrm{r}}$, resulting in an enhanced wettability of the solid surface. Interestingly, two different linear wetting regimes of water droplets on a gold substrate were observed and characterized by a critical value of Au-O interaction strength $\left(\varepsilon_{\mathrm{Au}-\mathrm{O} \text { critical }}\right)$. For $\varepsilon_{\mathrm{Au}-\mathrm{O}}<\varepsilon_{\mathrm{Au}-\mathrm{O} \text { critical }}$, the linear variation of the CA was steeper than in cases with $\varepsilon_{\mathrm{Au}-\mathrm{O}}>\varepsilon_{\mathrm{Au}-\mathrm{O} \text { critical }}$, where $\varepsilon_{\mathrm{Au}-\mathrm{O} \text { critical }}$ was calculated to be $2.2 \varepsilon_{\mathrm{F}-\mathrm{F}}$. In 2012 , Rafiee et al. experimentally and computationally measured the CA of water droplets on gold and copper substrates [46]. It was found that gold and copper surfaces resulted in $77.4^{\circ}$ and $85.92^{\circ}$ experimental wetting angles of water, respectively. We additionally defined the $\mathrm{Cu}$-water and $\mathrm{Au}$-water interaction strengths to recover the wetting behavior from the experiments. For instance, Rafiee and co-workers revealed that $\mathrm{MD}$ simulations in which $\varepsilon_{\mathrm{r}}^{\mathrm{Cu}-\mathrm{O}}$ was 1.1 showed an $84^{\circ}$ contact angle of the water droplet, demonstrating excellent reproducibility of the respective experimental results. However, a similar $\mathrm{Cu}-\mathrm{O}$ potential energy was used in this study, which exhibited a larger CA between the water droplet and copper substrate measuring $118.2^{\circ}$. Moreover, the stronger interaction strength between copper and water at $\varepsilon_{\mathrm{r}}=1.7$ induced a CA of $86.4^{\circ}$, which was consistent with the respective experimental values. We deduce that there are two origins for this disagreement: (1) The crystal structure of the Cu substrate, in which the $\mathrm{Cu}(001)$ planes were utilized to compose our $\mathrm{Cu}$ substrates. In contrast, the $\mathrm{Cu}$ walls in the report of Rafiee et al. were modeled by $\mathrm{Cu}$ (111) planes which were more dense and wetting than the $\mathrm{Cu}$ (001) planes. (2) Thermal vibrating walls were used in this study, compared to the fixed walls in the other study. In addition, the effects of molecular vibrations on the wetting behaviors were examined in Ref. [47]. This is because a fixed surface expels the surface roughness produced by the corrugations of vibrating molecules and creates "an atomistically flat surface" that is more hydrophilic than in the case with the vibrating wall. On the other hand, we found that 

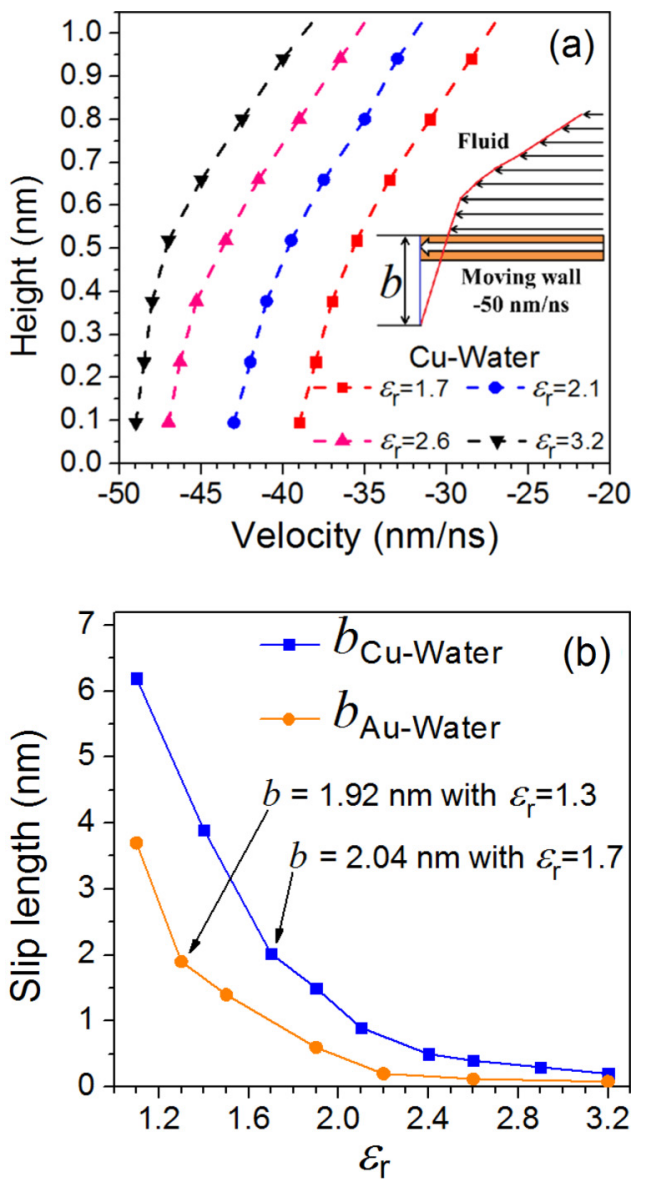

FIG. 3. (Color online) (a) Variation in velocity profiles according to the surface wettability in the vicinity of a solid wall; (b) slip length as a function of wall-fluid interaction strength ratio.

the experimental CA of water on a gold surface $\left(77.4^{\circ}\right)$ could be recovered by MD simulation when an interaction strength corresponding to $\varepsilon_{\mathrm{r}}^{\mathrm{Au}-\mathrm{O}}=1.3$ was utilized.

The next step was to compute the liquid-vapor surface tension of a water droplet on a solid substrate and the bulk viscosity of the water molecules in a periodic cubic box. Our computations showed that the surface tension released an average value of $72.062 \mathrm{mN} / \mathrm{m}$ at room temperature, which was consistent with other computations [48] and measurements [49]. Our findings additionally characterized the wall-fluid interaction and substrate material independence of the surface tension. Moreover, the accuracy in reproducing the surface tension value from experiments of the SPC-E water model by using MD simulations was demonstrated. In addition, the computed bulk viscosity of liquid water in a periodic cubic box was determined to be $0.67 \mathrm{cP}$, which yielded results compatible with those of both experiments [50] and MD simulations [51].

The last factor to be considered was the slip length at the solid-liquid interface, which was measured from the sheardriven flow model. Figure 3(a) shows the surface wettability dependence for the velocity profiles near the $\mathrm{Cu}$ wall obtained from a flow channel that was $4 \mathrm{~nm}$ in height using 30 slab bins. For the strong wetting cases $\left(\varepsilon_{\mathrm{r}}=2.6,3.2\right)$, the interface region showed similar velocities for the fluid and wall molecules.
This phenomenon implies that liquid water molecules were attached to the wall and moved with the wall molecules. Therefore, these liquid layers behaved as an extension of the wall layer. The inset in Fig. 3(a) is a schematic representation of the nanoscale steady-state fluid sheared between the solid surfaces. The slip length is the depth inside the molecular wall, in which the extrapolation of the velocity profile vanishes. Figure 3(b) is a plot of the slip length achieved from the cases studied in this paper. Our simulation results demonstrated that the slip length is highly dependent on $\varepsilon_{\mathrm{r}}$. An increase in $\varepsilon_{\mathrm{r}}$ enhances the momentum transfer at the boundary, resulting in a decreased slip length. Moreover, we found that cases of $\varepsilon_{\mathrm{r}}^{\mathrm{Cu} \text {-water }}=1.7$ and $\varepsilon_{\mathrm{r}}^{\mathrm{Au} \text {-water }}=1.3$ (demonstrating excellent recovery of the the experimental wetting behaviors of water on copper and gold substrates [46], respectively) resulted in slip lengths of 2.04 and $1.92 \mathrm{~nm}$ at the wall/fluid interface.

Thus far, we have investigated all the factors needed for calculation in the modified LW equation (2) with variations in the wall-fluid interaction strength. Additionally, we have obtained all of the data necessary to study the capillary rise of liquid water in nanotubes. However, it remains questionable whether Eq. (2) predicts the capillary imbibitions. We will discuss this in the next section.
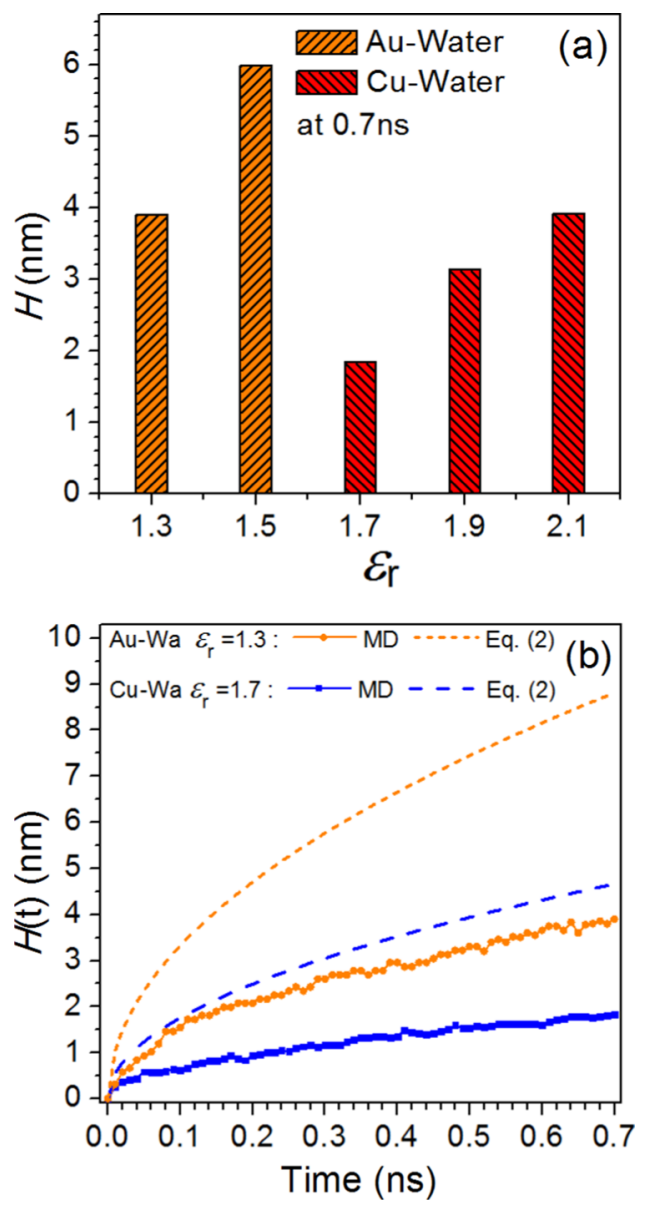

FIG. 4. (Color online) (a) Height of fluid meniscus inside nanoscale capillaries made of copper and gold at $0.7 \mathrm{~ns}$. (b) The uptake of liquid water into capillary for the cases $\varepsilon_{\mathrm{r}}^{\text {Cu-water }}=1.7$ and $\varepsilon_{\mathrm{r}}^{\mathrm{Au} \text {-water }}=1.3$ compared to the prediction of modified LW equation (2) at $0.7 \mathrm{~ns}$. 


\section{B. Nanoscale fluid rise in the cylindrical capillary and near-surface viscosity}

Figure 4(a) shows the height of the fluid meniscus inside the nanoscale gold and copper capillaries at $0.7 \mathrm{~ns}$ with the variation of $\varepsilon_{\mathrm{r}}$. From this figure, it is evident that the ability of capillary imbibitions is highly dependent on surface wetting. Therefore, the wettability of the capillary wall primarily defines efficient fluid entry into the tube. The momentum is transferred at the solid-liquid interface via intermolecular collisions and interactions. The more substantial the wetting of the capillary wall that is exhibited, the faster is the rate of fluid flow in the observed pore. An increase in $\varepsilon_{\mathrm{r}}$ improves the driving force for wetting, which leads to a higher length of imbibitions. Moreover, for the gold capillary cases with $\varepsilon_{\mathrm{r}}=1.3,1.5$, the fluid meniscus reached a higher level than in cases of a copper pore with $\varepsilon_{\mathrm{r}}=1.7,1.9,2.1$. These observations demonstrated the strength of the gold surfaces for capturing liquid molecules. Figure 4(b) represents the plots of the predictions from (2), as well as the MD-measured capillary imbibitions in the first $0.7 \mathrm{~ns}$ for the cases $\varepsilon_{\mathrm{r}}^{\mathrm{Au} \text {-water }}=1.3$ and $\varepsilon_{\mathrm{r}}^{\mathrm{Cu} \text {-water }}=1.7$. Our results confirmed that the height of the fluid meniscus is a function of the square root of time as $H(t) \propto \sqrt{t}$. Moreover, the MD fluid rise in the copper and gold nanopores was much slower than predicted from Eq. (2). A deviation between the height of the meniscus calculated from the MD simulations and the height predicted from (2) was observed to be significant at each point in the period of $0.7 \mathrm{~ns}$. Therefore, the factor that resists the capillary rise of liquid water to induce a significantly slower migration than (2) remains unclear.
In a nanoscale fluid flow, boundary length variations of a few molecular diameters significantly affect the characteristics of the flow region. The effects of the dynamic structuring on the fluid's viscosity are well known to resist the flows and have been demonstrated. Therefore, we conducted simulations to calculate the local viscosity of the liquid water sheared between the two solid walls. Figures 5(a) and 5(b) shows the stress tensor and density distributions of liquid water sheared between the copper and gold surfaces with $\varepsilon_{\mathrm{r}}^{\mathrm{Cu} \text {-water }}=1.7$ and $\varepsilon_{\mathrm{r}}^{\mathrm{Au} \text {-water }}=1.3$ along the channel height, respectively. To distinctly enable the observation of molecular distributions along the channel, the bin thickness must be ten times smaller than the molecular diameter [18]. Therefore, we divided our simulation domain into 100 slab bins with a corresponding width of $0.05644 \mathrm{~nm}$. This fine bin is not appropriate for a continuumlike analysis, but provides insight into the molecular structure in a similar manner to the radial distribution function. The density and stress tensor profiles also exhibit a similar tendency. Specifically, these properties fluctuated close to both the copper and gold surfaces, and converged on the bulk value in the mid-flow. Additionally, experimental evidence revealed that the density fluctuations are due to the effects of neighboring wall molecules [52]. Furthermore, the near-wall stress tensor points closer to the surface than $0.5 \mathrm{~nm}$, and showed fluctuations due to the local density variation of liquid water. The solid-liquid interface is defined as the region $0.5 \mathrm{~nm}$ from the solid wall. Therefore, the local viscosity is computed in each $0.5 \mathrm{~nm}$ region along the flow channel height based on (5).

Figure 5(c) shows the calculated local viscosity for the corresponding cases of $\varepsilon_{\mathrm{r}}$. It is clear that the dynamic
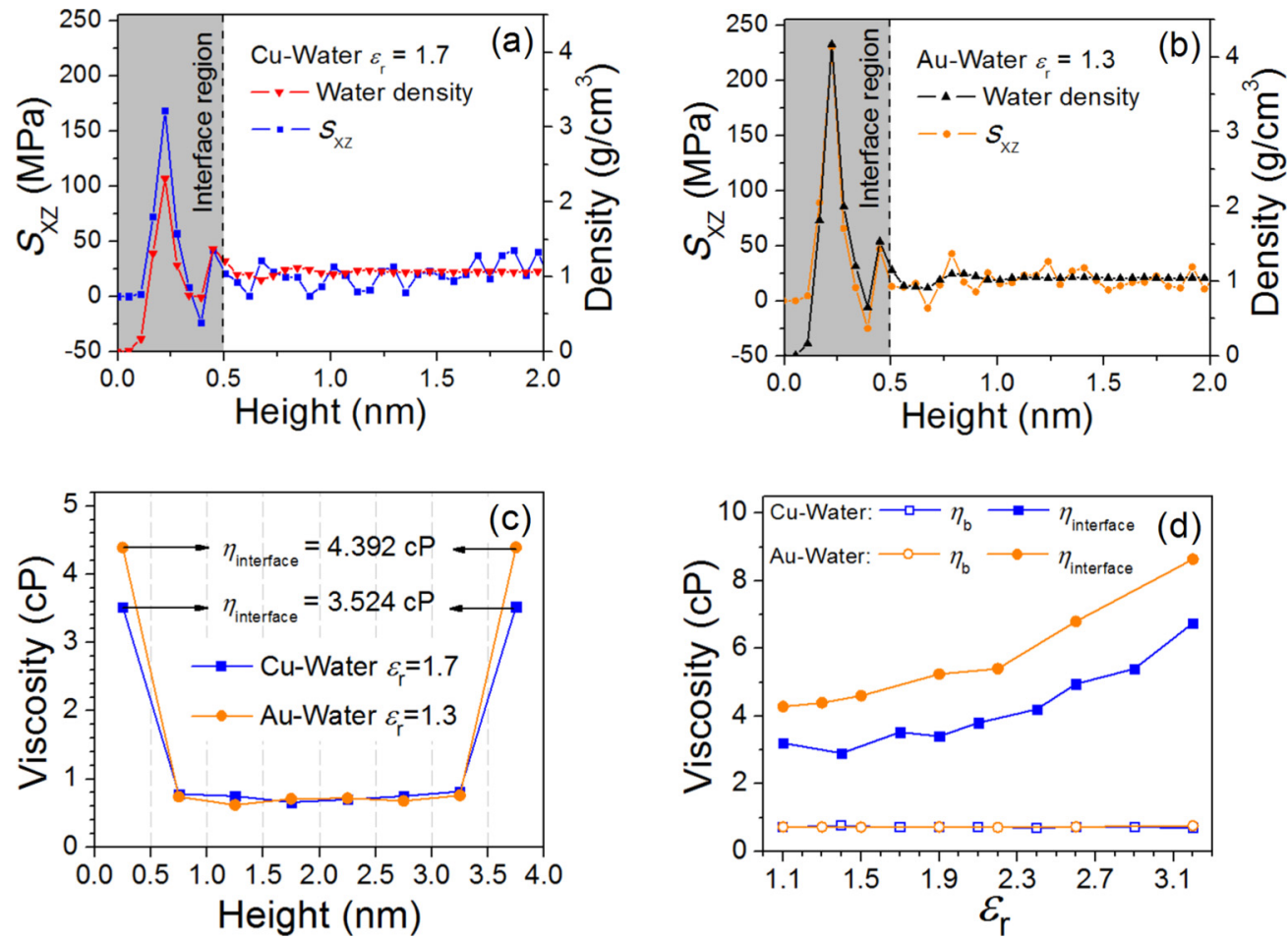

FIG. 5. (Color online) Shear stress $\left(S_{X Z}\right)$ and density distributions of liquid water sheared between solid walls presented together for the cases (a) $\varepsilon_{\mathrm{r}}^{\text {Cu-water }}=1.7$ and (b) $\varepsilon_{\mathrm{r}}^{\text {Au-water }}=1.3$. (c) The local viscosity profiles plotted for the corresponding cases of $\varepsilon_{\mathrm{r}}$. (d) The influence of wall-fluid interaction strength on the near-surface and bulk viscosity. 
structuring of liquid water, caused by the wall force penetration depth and interaction strength, induced a higher viscosity near the interface than in the middle of the fluid flow. $\varepsilon_{\mathrm{r}}^{\mathrm{Cu} \text {-water }}=1.7$ and $\varepsilon_{\mathrm{r}}^{\mathrm{Au} \text {-water }}=1.3$ resulted in $3.524 \mathrm{cP}$ and $4.392 \mathrm{cP}$ nearsurface viscosity, respectively. In contrast, the variation of the apparent and bulk viscosity with surface wettability is shown in Fig. 5(d). The results consistently demonstrate the differences between the near-wall and bulk values of viscosity with the increased wall-fluid potential energy. The viscosity of the liquid water in the middle of the shear-driven flow channel recovers the bulk viscosity of the liquid water molecules in a periodic cubic box and is independent of surface wettability. An average value of $0.69 \mathrm{cP}$ for viscosity in the mid-flow was achieved.

The effects of the surface force on the near-surface viscosity were examined. These influences were considered to constitute the primary reason for the slower MD migration of fluid into the nanoscale capillaries than in prediction (2). It is important to note that only the local viscosity near the surface differs from the others. Thus, the contribution of the bulk viscosity cannot be neglected. Hence, we define the effective viscosity $\left(\eta_{\text {eff }}\right)$ which considers the near-surface viscosity $\left(\eta_{\text {interface }}\right)$, the bulk viscosity $\left(\eta_{\text {bulk }}\right)$, and the conduit size. To effectively predict the capillary filling speed, $\eta_{\mathrm{eff}}$ is used to substitute for $\eta$ in Eq. (2). Rewriting (2) in terms of $\eta_{\text {eff }}$ yields

$$
H=\sqrt{\frac{\gamma_{\mathrm{LV}}(R+4 b) \cos \theta}{2 \eta_{\mathrm{eff}}}} \sqrt{t},
$$

where

$$
\begin{aligned}
& \eta_{\text {eff }}=\frac{R-L_{\text {interface }}}{R} \eta_{\text {bulk }}+\frac{L_{\text {interface }}}{R} \eta_{\text {interface }}, \\
& \eta_{\text {eff }}=\eta_{\text {bulk }}+\frac{L_{\text {interface }}}{R}\left(\eta_{\text {interface }}-\eta_{\text {bulk }}\right),
\end{aligned}
$$

and $L_{\text {interface }}$ is defined as the thickness of the solid-liquid interface, corresponding to $0.5 \mathrm{~nm}$ for this study. For $R \rightarrow \infty$, the fluid's effective viscosity is equal to the bulk viscosity, and the slip length does not exist.

Figure 6 displays the ratio of the height of the fluid meniscus derived from the predictions (2) and (8) to the MD-measured fluid rise at $0.7 \mathrm{~ns}$. The red dashed line signifies that the ratio is equal to 1 , signifying that the MD fluid rise and prediction are a $100 \%$ match. If the ratio is smaller than 1 , this implies that the theoretical prediction underestimates the MD fluid rise into the capillary. In contrast, if the ratio is greater than 1 , then the fluid rise from the prediction is faster than in MD simulations. Our results revealed that in Eq. (2) using bulk viscosity, there was a significant deviation for the MD fluid rise. However, a more accurate prediction of the MD fluid rise was obtained for both copper and gold capillary imbibitions when our approach (8) was used. Additionally, the cases $\varepsilon_{\mathrm{r}}^{\text {Au-water }}=1.3$ and $\varepsilon_{\mathrm{r}}^{\text {Cu-water }}=1.7$ adequately recovered the contact angle from experimental works and demonstrated excellent consistency with Eq. (8). Therefore, we believe that the near-surface viscosity and Navier slip length are two factors that must be accounted for when using the LW theory to predict the nanoscale fluid increase into capillaries.

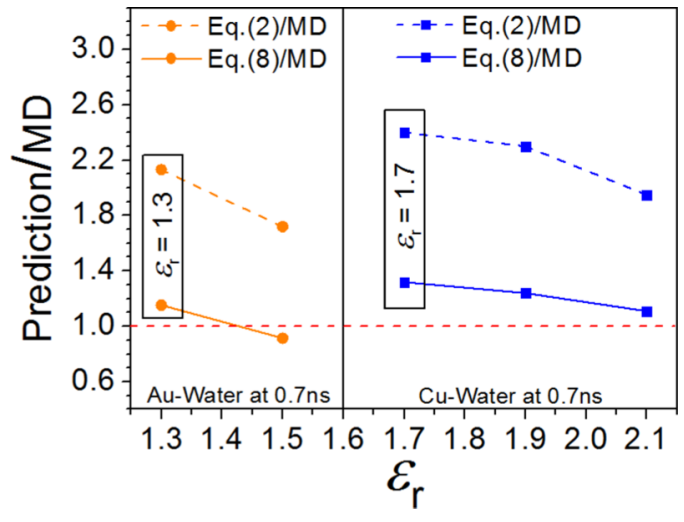

FIG. 6. (Color online) The ratio of the height of the fluid meniscus derived from the theoretical predictions (2) and (8) to the MD-measured fluid rise at $0.7 \mathrm{~ns}$ for several cases of surface wettability. The dashed red line denotes the height ratio for theory and MD meniscus results, in which the MD fluid rise and prediction are entirely matched.

\section{CONCLUSION}

The present study is dedicated to developing a theoretical tool for prediction of nanocapillary liquid rise. First, we investigated the validity of the modified LW equation (2) proposed for the liquid rise at small-scale conduits. For such a case, all of the parameters employed in Eq. (2) were properly calculated by an extensive series of molecular dynamics simulations. Prior to our MD simulations, we investigated the proper interaction strengths for the water-gold and watercopper couples studied here. Our water droplet simulations showed that $\varepsilon_{\mathrm{r}}^{\mathrm{Au} \text {-water }}=1.3$ and $\varepsilon_{\mathrm{r}}^{\mathrm{Cu} \text {-water }}=1.7$ adequately reproduced the experimental contact angle of a water droplet on copper $\left(85.92^{\circ}\right)$ and gold $\left(77.4^{\circ}\right)$ surfaces, respectively. Molecular dynamics simulations employing the determined proper interaction strengths were further used to calculate the liquid-vapor surface tension, the bulk viscosity, and the slip length at solid-liquid interfaces. Using the molecularly calculated parameters, Eq. (2) showed significant difference from the experimental MD fluid rise into nanocapillaries. Slower MD capillary imbibitions were observed for both gold and copper tubes compared to the prediction of Eq. (2). This controversy is developed since the proposed extension of the LW equation considers a Navier-type slip length only in order to include small-scale effects. However, nanoscales develop further complications. The wall-fluid interaction forces dynamically attract liquid water molecules to the solid surface and induce a structured liquid layering with a higher density and viscosity than the conduit center. In this capacity, these liquid layers function as an extended wall layer. In a nanoscale fluid flow, variations in such a small region extending a few molecular diameters predominantly influence the flow behaviors. In order to understand the role of the apparent viscosity, the local viscosity of the water was computed. It was found that the near-surface viscosity is considerably larger than the viscosity in the mid-flow due to the influences of the solid surface. This near-surface viscosity is proposed to be the primary reason for the resistance of the MD fluid flow into the nanopores, resulting in a slower entry than the predictions of Eq. (2). Therefore, the effects of both the near-surface 
viscosity and slip length should be considered for an accurate nanocapillary liquid rise calculation. Our approach given in Eq. (8) demonstrated good agreement with the MD-measured capillary rise of the liquid water in nanopores.

\section{ACKNOWLEDGMENT}

This work was supported by the 2015 Research Fund of the University of Ulsan (Republic of Korea).
[1] C. Rivet, H. Lee, A. Hirsch, S. Hamilton, and H. Lu, Chem. Eng. Sci. 66, 1490 (2011).

[2] M. A. Nilsson, R. Kulkarni, L. Gerberich, R. Hammond, R. Singh, E. Baumhoff, and J. P. Rothstein, J. Non-Newtonian Fluid Mech. 202, 112 (2013).

[3] Y. Li, J. Wang, M. G. Wientjes, and J. L. Au, Adv. Drug Delivery Rev. 64, 29 (2012).

[4] N. Tas, J. Haneveld, H. Jansen, M. Elwenspoek, and A. Van Den Berg, Appl. Phys. Lett. 85, 3274 (2004).

[5] R. Lucas, Kolloid Z. 23, 15 (1918).

[6] E. W. Washburn, Phys. Rev. 17, 273 (1921).

[7] L. Bocquet and E. Charlaix, Chem. Soc. Rev. 39, 1073 (2010).

[8] D. I. Dimitrov, A. Milchev, and K. Binder, Phys. Rev. Lett. 99, 054501 (2007).

[9] C. Cupelli, B. Henrich, T. Glatzel, R. Zengerle, M. Moseler, and M. Santer, New J. Phys. 10, 043009 (2008).

[10] C. Chen, C. Gao, L. Zhuang, X. Li, P. Wu, J. Dong, and J. Lu, Langmuir 26, 9533 (2010).

[11] G. Martic, F. Gentner, D. Seveno, D. Coulon, J. De Coninck, and T. Blake, Langmuir 18, 7971 (2002).

[12] M. R. Stukan, P. Ligneul, J. P. Crawshaw, and E. S. Boek, Langmuir 26, 13342 (2010).

[13] L. Joly, J. Chem. Phys. 135, 214705 (2011).

[14] D. Schebarchov and S. C. Hendy, Phys. Rev. E 78, 046309 (2008).

[15] N. V. Priezjev, A. A. Darhuber, and S. M. Troian, Phys Rev E. 71, 041608 (2005).

[16] N. Asproulis and D. Drikakis, Phys. Rev. E 84, 031504 (2011).

[17] B. Kim, Chem. Phys. Lett. 554, 77 (2012).

[18] B. H. Kim, A. Beskok, and T. Cagin, Microfluid. Nanofluid. 9, 31 (2010).

[19] T. Vo, B. Park, C. Park, and B. Kim, J. Mech. Sci. Technol. 29, 1681 (2015).

[20] J.-L. Barrat and L. Bocquet, Phys. Rev. Lett. 82, 4671 (1999).

[21] B. H. Kim, A. Beskok, and T. Cagin, J. Chem. Phys. 129, 174701 (2008).

[22] B. H. Kim, A. Beskok, and T. Cagin, Microfluid. Nanofluid. 5, 551 (2008).

[23] N. Asproulis and D. Drikakis, Phys. Rev. E 81, 061503 (2010).

[24] J. Sun, Y. He, W. Tao, X. Yin, and H. Wang, Int. J. Numer. Methods Eng. 89, 2 (2012).

[25] M. Kalweit and D. Drikakis, J. Comput. Theor. Nanosci. 5, 1923 (2008).

[26] G. R. Willmott and J. L. Tallon, Phys. Rev. E 76, 066306 (2007).
[27] A. Hibara, T. Saito, H. Kim, M. Tokeshi, T. Ooi, M. Nakao, and T. Kitamori, Anal. Chem. 74, 6170 (2002).

[28] D. Burgreen and F. Nakache, J. Phys. Chem. 68, 1084 (1964).

[29] N. Churaev, V. Sobolev, and Z. Zorin, Special Discussion on Thin Liquid Films and Boundary Layers (Academic, New York, 1971), pp. 213-220.

[30] J. Ryckaert, G. Ciccotti, and H. J. Berendsen, J. Comput. Phys. 23, 327 (1977).

[31] H. Berendsen, J. Grigera, and T. Straatsma, J. Phys. Chem. 91, 6269 (1987).

[32] M. P. Allen and D. J. Tildesley, Computer Simulation of Liquids (Clarendon Press, Oxford, 1989), p. 385.

[33] J. Mei, J. W. Davenport, and G. W. Fernando, Phys. Rev. B 43, 4653 (1991).

[34] R. D. Groot and P. B. Warren, J. Chem. Phys. 107, 4423 (1997).

[35] W. Stroberg, S. Keten, and W. K. Liu, Langmuir 28, 14488 (2012).

[36] T. Soddemann, B. Dünweg, and K. Kremer, Phys. Rev. E 68, 046702 (2003).

[37] S. Plimpton, J. Comput. Phys. 117, 1 (1995).

[38] J. Lopez-Lemus and J. Alejandre, Mol. Phys. 100, 2983 (2002).

[39] B. Hess, J. Chem. Phys. 116, 209 (2002).

[40] P. Xu, T. Cagin, and W. A. Goddard III, J. Chem. Phys. 123, 104506 (2005).

[41] J. Koplik, J. R. Banavar, and J. F. Willemsen, Phys. Fluids A 1, 781 (1989).

[42] M. Barisik and A. Beskok, Microfluid. Nanofluid. 11, 269 (2011).

[43] H. Heinz, W. Paul, and K. Binder, Phys. Rev. E 72, 066704 (2005).

[44] A. P. Thompson, S. J. Plimpton, and W. Mattson, J. Chem. Phys. 131, 154107 (2009).

[45] P. A. Thompson and S. M. Troian, Nature (London) 389, 360 (1997).

[46] J. Rafiee, X. Mi, H. Gullapalli, A. V. Thomas, F. Yavari, Y. Shi, P. M. Ajayan, and N. A. Koratkar, Nat. Mater. 11, 217 (2012).

[47] M. Barisik and A. Beskok, Mol. Simul. 39, 700 (2013).

[48] J. Alejandre, D. J. Tildesley, and G. A. Chapela, J. Chem. Phys. 102, 4574 (1995).

[49] R. Breslow and T. Guo, Proc. Natl. Acad. Sci. USA 87, 167 (1990).

[50] K. R. Harris and L. A. Woolf, J. Chem. Eng. Data 49, 1064 (2004).

[51] S. Tazi, A. Boţan, M. Salanne, V. Marry, P. Turq, and B. Rotenberg, J. Phys.: Condens. Matter 24, 284117 (2012).

[52] F. Heslot, N. Fraysse, and A. Cazabat, Nature (London) 338, 640 (1989). 